\title{
Eficacia de un programa de prevención cognitivo-conductual breve del rechazo social en niños
}

\author{
Effectiveness of a brief cognitive-behavioral prevention program for social rejection in \\ children
}

\author{
Ornela Mateu-Martínez \\ José Antonio Piqueras \\ $\mathrm{M}^{\mathrm{a}}$ Isabel Jiménez-Albiar \\ José Pedro Espada \\ José Luis Carballo \\ Mireia Orgilés
}

Departamento de Psicología de la Salud, Universidad Miguel Hernández de Elche, Elche, España.

(Rec: 25 de junio de 2012 / Acep: 30 de enero de 2013)

\section{Resumen}

Los programas de prevención del rechazo social en la infancia son todavía muy escasos. El objetivo de este estudio fue evaluar los efectos de un programa de intervención cognitivo-conductual breve basado en el desarrollo de competencias socio-emocionales, diseñado para prevenir el rechazo social infantil y patologías asociadas. Para ello se siguió un diseño cuasiexperimental pretest-postest con grupo control no equivalente. La muestra se compuso de 94 estudiantes de 8 a 12 años. El tratamiento consistió en un programa multicomponente cognitivo-conductual aplicado en grupo, con una frecuencia semanal y un total de 6 sesiones de 60 minutos cada una. Los resultados apoyaron la eficacia del programa de desarrollo de competencias infantil, obteniendo efectos positivos en las medidas de ansiedad, depresión, inteligencia emocional y estatus social del sociograma. Estos datos muestran la eficacia y necesidad de prevenir la aparición del rechazo social y patologías asociadas en la infancia en el contexto escolar.

Palabras clave: rechazo social, niños, prevención, cognitivo-conductual, Inteligencia Emocional.

\begin{abstract}
Prevention programs for social rejection in children are still scarce. The aim of this study was to determine the effectiveness of a cognitive-behavioral intervention program, based on development of socio-emotional competencies, designed to prevent child social rejection and associated diseases. We followed a pretest-posttest non-equivalent control group quasi-experimental design. The sample consisted of 94 students between 8 and 12 years. Treatment consisted of a multicomponent cognitive-behavioral program, administered on a group basis, with one weekly session lasting 60 minutes each one, for a total of 6 sessions. The results supported the effectiveness of skill development program for children, showing positive treatment effects on measures of anxiety, depression, emotional intelligence and social status by sociogram. These data show the effectiveness and need of preventing the emergence of childhood social rejection and associated diseases in school setting. Key words: Social rejection, children, prevention, cognitive-behavioral, Emotional Intelligence.
\end{abstract}

Correspondencia: José Ant ${ }^{\circ}$ Piqueras, Departamento de Psicología de la Salud, Universidad Miguel Hernández de Elche, Edificio Altamira, Avda. de la Universidad, s/n Edf. Altamira, 03202 Elche (España) Correo-e: jpiqueras@umh.es 


\section{Introducción}

La necesidad de pertenencia a un grupo es una fuerte motivación en el ser humano y se ha mantenido a lo largo de la evolución dado que aumenta la tasa de supervivencia de los individuos. Por esta razón, la exclusión social produce fuertes sentimientos negativos en la persona que lo padece (Magallares, 2011). Además, las relaciones con los iguales juegan un papel importante en el desarrollo general del ser humano (Inglés et al., 2009). En este proceso, la mayoría de niños establecen relaciones satisfactorias, aunque algunos se ven apartados y/o no disfrutan de esas interacciones padeciendo rechazo social (García-Bacete, Monjas y Sureda, 2010).

Respecto al primero de los escenarios, la aceptación social, esta es considerada como el grado en que un niño es querido, aceptado, reconocido y apreciado en su grupo de iguales (Bukowski, Newcomb y Hartup, 1996). La aceptación social y las buenas relaciones sociales están asociadas con una buena adaptación, bienestar personal, mayor rendimiento escolar, alta autoestima y un sentimiento positivo y placentero dentro del grupo (Wentzel, 2003). En este mismo sentido, los amigos son elementos importantes de compañía, diversión, protección, información y aprendizaje, fuente de intimidad, afecto, seguridad y apoyo emocional. Tener amigos es un indicador de buenas habilidades interpersonales y un signo de un buen ajuste psicológico posterior (Inglés, Delgado, García-Fernández, Ruíz-Esteban y Díaz-Herrero, 2010).

La condición contraria es el rechazo social. Esta es una de las causas del no acceso a unas buenas relaciones. Se relaciona con otros fenómenos tales como bullying, acoso, aislamiento, violencia, maltrato, exclusión o marginación. Para Asher y Paquette (2003) el rechazo por parte de los iguales conduce al sentimiento de soledad dentro del grupo, pobre aceptación, falta de amistades duraderas y de calidad, y el llegar a ser víctima en situaciones de acoso escolar. Así, la baja aceptación social, la ignorancia y el rechazo son factores de riesgo importantes para la desadaptación (Bukowski et al., 1996). Estudios recientes han hallado que los adolescentes implicados como agresores y/o víctimas muestran el perfil psicosocial menos adaptativo en términos de autoestima general, satisfacción con la vida, sintomatología depresiva, reputación, idea no conformista e implicacion en la comunidad (Povedano, Estévez, Martínez y Monreal, 2012). Según Gifford-Smith y Brownell (2003), los rechazados aparecen como el grupo sociométrico con mayor riesgo en su desarrollo. Así, numerosos estudios coinciden en señalar que, si bien no hay un perfil prototípico del alumno rechazado, la mayoría de estos muestran alguno o varios de los siguientes patrones de conducta: a) bajas tasas de conductas prosociales; $b$ ) altas tasas de conductas agresivas o disruptivas; c) altas tasas de conductas de falta de atención y/o inmaduras; d) altas tasas de conductas de ansiedad y evitación; e) escaso nivel de actividad social; f) menor autoestima y depresión; g) disfrutan menos de las actividades de clase, se muestran insatisfechos con las relaciones-ayudas de profesores y compañeros y con la claridad-coherencia-penalización de las normas de clase; además de presentar dificultades de aprendizaje y predecir mal rendimiento académico; h) perciben a sus familias como menos cohesionadas, más conflictivas, con menor grado de comunicación positiva y orientación al logro y de planificación y participación en actividades culturales (Bierman, 2004; Eshel, Sharabany y Barsade, 2003; GarcíaBacete, Monjas y Sureda, 2008; Plazas et al., 2010; Zhu, Xu y Kong, 2000). Las consecuencias son graves, destacando desajuste y abandono escolar, delincuencia, sentimientos de ansiedad, depresión y un riesgo aumentado de continuar con problemas psiquiátricos en la vida adulta (Campbell et al., 2006).

Desde los años 60 los estudios han reflejado que en el aula tradicional se producían frecuentes y complejos procesos de discriminación entre iguales, que es necesario conocer para poder prevenir y que el profesorado disponga de recursos eficaces para adaptar la enseñanza a la diversidad de los alumnos y comprometerse con la construcción activa de la igualdad y con la lucha contra la exclusión (Díaz-Aguado, 2002). Muñoz (2007) señala que en la actualidad el rechazo se da prácticamente en el $100 \%$ de las aulas, porque es un fenómeno muy ligado a la vida de los grupos. En torno a un $10 \%$ de niños son rechazados en cada aula, aunque con distinta manifestación. El rechazo no parte del individuo, sino del grupo, restando oportunidades de aprendizaje social, mermando la autoconfianza, creando sentimientos negativos, cronificando la situación de los niños rechazados y potenciando los problemas de conducta. El rechazo es un fenómeno complejo que requiere una intervención compleja y supone trabajar de forma conjunta en dos líneas principales: evitar/disminuir el rechazo y promover la aceptación.

Rajmil et al. (2009) señalan que el acoso más fuerte se produce en edades muy tempranas (entre 8 y 11 años), de modo que las políticas de prevención y tratamiento deberían también adelantarse. España se encuentra entre los cinco países analizados donde los niños tienen más probabilidades de sentirse víctimas de bullying, está por encima de la media, con una probabilidad entre un $22 \%$ y un $23 \%$. Según García-Bacete et al. (2008) en torno al 10$15 \%$ de los niños en el contexto escolar son rechazados por 
sus compañeros. En las investigaciones se ha encontrado una media de $11.3 \%$ de niños rechazados, datos similares a otras investigaciones nacionales e internacionales. Según McKown (2010) casi el 13\% de la población escolar tiene dificultades de aprendizaje socio-emocional. La habilidad de los niños para desarrollar relaciones positivas con sus compañeros es crítica para su bienestar.

Las competencias sociales son un aspecto fundamental de las habilidades humanas (Yugar y Shapiro, 2001) y un importante indicador de ajuste (Hussong, 2000). Especialmente importantes en el desarrollo de las competencias sociales son las relaciones que se establecen con los pares durante la escolarización, cuando el niño pasa de la dependencia total del hogar a su segundo sistema de socialización, donde comenzará a compartir con sus compañeros y a ser influenciado e influir en el comportamiento de ellos. Así, la empatía, uno de los aspectos de la competencia socioemocional, se considera un factor de protección contra el desarrollo de la conducta antisocial (Mestre, Samper y Frías, 2004).

Según García-Bacete et al. (2008) no todas las intervenciones realizadas han mostrado ser eficaces, por ello urge el estudio y el diseño de procedimientos fiables de identificación y de promoción de interacciones positivas entre los alumnos. Ha habido algunos intentos por intervenir en el contexto escolar para mejorar estos problemas (por ejemplo, Fernández-Cabezas, Benítez, Fernández, Justicia y Justicia-Arráez, 2011), si bien la mayoría más centrados en fenómenos relacionados tales como la agresividad y la delincuencia escolar (Guerra, Campaña, Fredes, Gutiérrez y Plaza, 2011, Livacic-Rojas, Espinoza y Ugalde, 2004; Pérez, Fernández, Rodríguez y de la Barra, 2005). La mayoría de ellos aconseja poner en práctica programas de enseñanza de habilidades sociales en poblaciones cuando se presentan los primeros síntomas de conducta antisocial o incluso con estudiantes con problemas persistentes de conducta (Fernández et al., 2011; Guerra et al., 2011; Knut y Frode, 2005; Livacic-Rojas et al., 2004; Pérez et al., 2005). Sin embargo hay una serie de limitaciones en estos estudios: las intervenciones se centran en la intervención secundaria más que primaria, los programas se han desarrollado generalmente para adolescentes, las intervenciones en el ámbito escolar se llevan a cabo por docentes con escasa formación especializada, y además hay escasos programas de desarrollo de competencias sociales e Inteligencia Emocional en educación primaria en nuestro país, especialmente llevados a cabo por profesionales especializados en el contexto escolar.

En consecuencia, el objetivo de este trabajo es evaluar mediante un estudio piloto la eficacia de un programa de prevención del rechazo social y mejora de las relaciones basado en el desarrollo de competencias sociales e Inteligencia Emocional, con el fin de mejorar las relaciones entre iguales en alumnos de Educación Primaria, y servir como método de prevención del rechazo social y las psicopatologías asociadas.

Con el fin de aportar evidencia sobre la eficacia de este programa de prevención breve se hipotetizó que: (1) el grupo experimental y el grupo control serían equivalentes estadísticamente en el pretest; (2) no se hallarían cambios significativos entre las medidas en el pretest y el postest en el grupo control; (3) se produciría una mejora significativa en las variables consideradas entre pretest y postest en el grupo de tratamiento; y (4) habría diferencias estadísticamente significativas entre grupo de tratamiento y grupo control en el postest, a favor del primero.

\section{Método}

\section{Participantes}

El total de sujetos reclutados fue de 130 estudiantes de $3^{\circ}$ a $6^{\circ}$ de Educación Primaria, de los que 34 (26.15\%) fueron excluidos por errores u omisiones en sus respuestas, por no obtener el consentimiento de los padres para participar o por presentar trastornos de aprendizaje. La muestra definitiva se compuso de 94 estudiantes distribuidos en dos grupos: i) grupo control: 27 alumnos de $3^{\circ}$ a $6^{\circ}$ curso con un rango de edad de 8 a 12 años $(M=9.74 ; D T=.712)$ y ii) grupo experimental 67 alumnos de $3^{\circ}$ a $6^{\circ}$ curso, con un rango de edad de 8 a 12 años $(M=9.76 ; D T=1.22)$.

La prueba chi-cuadrado de homogeneidad de la distribución de frecuencias reveló la ausencia de diferencias estadísticamente significativas en el grupo experimental en la asociación entre género y edad $(\chi 2=1.617 ; p=.89)$. Lo mismo se halló en el grupo control en género y edad $(\chi 2$ $=1.105 ; p=.59$ ). Por tanto no hubo interdependencia o asociación sexo por edad en ninguno de los grupos.

\section{Instrumentos}

Se emplearon las mismas pruebas en el pretest y en el postest.

La integración social se evaluó mediante dos cuestionarios sociométricos: el «Cuestionario Sociométrico para Niños (CSN)» (Díaz-Aguado, 1995), de 6 a 10 años para segundo ciclo ( $3^{\circ}$ y $4^{\circ}$ de Primaria). Para tercer ciclo $\left(5^{\circ}\right.$ y $6^{\circ}$ de Primaria) se utilizó el «Cuestionario Sociométrico para Preadolescentes (CSP)», de 11 a 13 años (Díaz-Aguado, 1995). Evalúa la integración social de los alumnos en sus 
aulas. El tiempo estimado de aplicación es de 5 minutos. Se basa en el método de las nominaciones: se pide al alumno que nombre a los tres chicos o chicas de su grupo con los que más le gusta jugar o estar en su tiempo libre y con los que menos le gusta jugar o estar, preguntándole el porqué de sus elecciones y rechazos. Se calcula un índice de rechazos: entre el número de rechazos posibles y el total de alumnos. Del mismo modo se obtiene el índice de elecciones.

Para valorar la Inteligencia Emocional se empleó el «Test de Inteligencia Social Emocional» (Chiriboga y Franco, 2001). Consta de 60 ítems, doce para cada escala: autoconciencia, autocontrol, automotivación o aprovechamiento emocional, empatía y habilidad social. Esta prueba presenta una Sensibilidad del $45.45 \%$, una Especificidad del $75.95 \%$. Tiene valores predictivos positivo y negativo de 34.48 y $83.33 \%$, respectivamente, para un Valor Predictivo Global igual a 69.1\% (Chiriboga y Franco, 2001). El tiempo estimado de aplicación es de 30 minutos. La escala de respuesta es tipo Likert con las posibilidades de nunca (0), a veces (1), casi siempre (2) y siempre (3). La interpretación de las puntuaciones sugiere que a mayor puntuación, mayor Inteligencia Emocional presenta el sujeto. Los valores alfa de Cronbach de las puntuaciones en esta muestra fueron: puntuación total $=.83$, Autoconciencia $=.53$, Autocontrol $=.54$, Aprovechamiento emocional $=.62$, Empatía $=.63$, Habilidad social $=.71$ ).

Se empleó el «Inventario de Depresión Infantil» (Children's Depresión Inventory, CDI, Kovacs, 1992), versión adaptada por Del Barrio, Moreno-Rosset y López-Martínez (1999) a población española, que es el más utilizado para evaluar la existencia de sintomatología depresiva (Herrera, Núñez, Tobón y Arias, 2009). La fiabilidad del CDI es elevada, con una sensibilidad de 94.7\%, una especificidad del 95.6\%, un valor predictivo positivo de $0.90 \mathrm{y}$ un valor predictivo negativo de 0.98 (Figueras, Amador-Campos, Gómez-Benito y del Barrio, 2010). Consta de 27 ítems estructurados en dos escalas: Disforia y Autoestima. El tiempo estimado de aplicación es de 10 minutos. Las opciones de respuesta siguen una escala tipo Likert que va desde 0 a 2. La interpretación sugiere que a mayor puntuación, mayor sintomatología depresiva presenta el sujeto. Los valores alfa de Cronbach de las puntuaciones en esta muestra fueron: Disforia $=.80$, Autoestima $=.68$ y puntuación total $=84$.

Para evaluar la existencia de sintomatología ansiosa, se utilizó la «Escala de Ansiedad Infantil de Spence» (Spence Children's Anxiety Scale SCAS, Spence, 1998), versión española europea de Orgilés (2009), que es una prueba que puede utilizarse con confianza en la evaluación de los problemas de ansiedad infanto-juveniles. Las propiedades psicométricas de la escala con población española son buenas, con una alta consistencia interna (alpha de Cronbach $=.89)$ y una validez convergente $(r=.41)$ adecuada con otra medida de ansiedad (Orgilés, Méndez, Spence, HuedoMedina, Espada, 2012). Consta de 45 ítems distribuidos en 6 subescalas: Ansiedad por separación, Fobia social, Trastorno obsesivo-compulsivo, Pánico/Agorafobia, Miedo al daño físico, Ansiedad generalizada. El tiempo estimado de aplicación es de 30 minutos. Las respuestas se puntúan en una escala tipo Likert que va desde nunca (0), a veces (1), muchas veces (2) y siempre (3). La interpretación sugiere que a mayor puntuación, mayor sintomatología ansiosa presenta el sujeto. Los valores alfa de Cronbach de las puntuaciones en esta muestra fueron: Trastorno de Ansiedad Separación $=.58$, Fobia Social $=.69$, Trastorno Obsesivo-compulsivo $=.56$, Pánico/Agorafobia $=.80$, Daño físico $=.54$, Trastorno de Ansiedad Generalizada $=$ .62 y puntuación total $=.86$.

\section{Procedimiento}

Se llevó a cabo una entrevista con los directores de los centros que accedieron a participar en la investigación para exponer los objetivos de esta, describir los instrumentos de evaluación, solicitar permiso y promover su colaboración. Se seleccionó a los alumnos de dos colegios públicos de Educación Primaria de la provincia de Alicante. Se solicitó a los padres el consentimiento informado por escrito autorizando a sus hijos a participar en la investigación.

Se llevó a cabo un diseño cuasi-experimental pretestpostest con grupo control no equivalente, asignando los sujetos por conveniencia a control y tratamiento en función del centro (Ramos, 2011). La evaluación se realizó de igual forma en ambos grupos, los cuestionarios se aplicaron el mismo día en horario de clase, de forma colectiva y voluntaria, asignando a cada alumno un cuaderno de evaluación con las hojas de respuesta de cada prueba. Se indicó que cumplimentaran los datos de identificación (nombre y apellidos, edad, fecha de nacimiento, curso, género, fecha, centro y país de nacimiento) y se leyeron en voz alta las instrucciones, resaltando la importancia de no dejar preguntas sin contestar. Los investigadores estuvieron presentes durante la administración de las pruebas para aclarar dudas y verificar la cumplimentación independiente por parte de los sujetos. El tiempo medio de aplicación de la batería de pruebas fue de 60 minutos. 


\section{Programa de intervención}

Se diseñó un programa basado en el desarrollo de competencias sociales e Inteligencia Emocional, que combina técnicas cognitivo-conductuales. Consistió en 6 sesiones de 60 minutos de duración, con una frecuencia semanal, que se realizaron con todo el grupo en el aula. Las técnicas utilizadas fueron: resolución de problemas, reestructuración cognitiva, modelado, autocontrol, entrenamiento en habilidades sociales (habilidades de comunicación y asertividad), técnicas operantes para la adquisición y mantenimiento de conductas (reforzamiento positivo social), técnicas de control de la activación (respiración diafragmática y relajación muscular progresiva). La estructuración de las sesiones, objetivos, contenidos y desarrollo de cada una de ellas se describe en la tabla 1.

Tabla 1. Sesiones, objetivos, y contenidos de las sesiones en la aplicación del programa cognitivo-conductual para favorecer las relaciones y prevenir el rechazo social.

\section{SESIÓN 1}

Objetivos

- Introducción de la organización y estructura del programa

- Conocer la importancia de la comunicación

- Identificar los estilos comunicativos

- Fomentar las habilidades de comunicación y asertividad

Contenido

- Presentación e introducción del programa, la importancia de la comunicación, identificar los estilos comunicativos y estrategias que ayuden a ser más asertivos

\section{SESIÓN 2}

Objetivos

- Comprender e identificar nuestras emociones y sus efectos

- Expresión y manejo de las emociones

- Aprender a reconocerlas e interpretarlas en otros

Contenido

- Las emociones primarias y secundarias, su función en la adaptación, cómo expresarlas, identificarlas e interpretarlas en los demás.

\section{SESIÓN 3}

Objetivos

- Entrenar en técnicas de relajación y respiración para afrontar el estrés, tensiones y dificultades

Contenido

- Técnica de relajación muscular progresiva, técnica de respiración diafragmática, fantasía dirigida

\section{SESIÓN 4}

Objetivos

- Entrenar en la resolución de problemas, en previsión y anticipación de consecuencias y en toma de decisiones

- Potenciar la empatía

Contenido

- Solución de problemas en situaciones de la vida cotidiana en diferentes contextos (personal, social, escolar, familiar) siguiendo los pasos del modelo propuesto

\section{SESIÓN 5}

Objetivos

- Fomentar el autocontrol personal, la autorregulación, reforzar comportamiento adecuado y ofrecer retroalimentación sobre el proceso de solución de problemas.

Contenido

- Técnicas de autocontrol, autoinstrucciones, solución de problemas y toma de decisiones

\section{SESIÓN 6}

Objetivos

- Favorecer el desarrollo de la autoestima y el sentimiento de identidad propia

- Afianzar los contenidos aprendidos en las sesiones anteriores y resolver dudas

- Valorar aspectos del programa

Contenido

- Cómo se construye la autoestima, importancia de la autoimagen y heteroimagen, reestructuración cognitiva en distorsiones de pensamiento, definirse a sí mismo y potenciar aspectos positivos. Revisión de las estrategias aprendidas durante el programa 


\section{Análisis de datos}

Los datos fueron codificados y analizados con el IBM SPSS 18.0. Con la finalidad de evaluar el cambio en las variables objeto de estudio se obtuvieron las medias y las desviaciones típicas de cada variable en los sujetos experimentales y control, en la fase pretest y postest. El nivel de confianza utilizado fue del $95 \%$.

Se realizaron análisis para comprobar la normalidad de las puntuaciones y comprobar si procedía aplicar pruebas paramétricas o no, siendo normales en su mayoría. Se aplicó la prueba de Rachas para comprobar si los datos se distribuían aleatoriamente. Se utilizó el principio de Levene de homogeneidad de varianzas. Mediante la prueba T-Student se realizó el análisis de la equivalencia de la puntuaciones en el pretest entre grupo experimental y control, análisis pretest-postest en el grupo control para garantizar la ausencia de variables extrañas que han contaminado la investigación, análisis pretest-postest en el grupo experimental y por último análisis del postest (diferencias entre grupo control y experimental en el postest).

Se incluyó el índice $d$ (diferencia media tipificada) propuesto por Cohen (1988), que permite valorar la magnitud o el tamaño del efecto de las diferencias halladas. Su interpretación es: tamaño del efecto pequeño (0.20 - 0.49), mediano $(0.50-0.79)$ y grande $(d \geq 0.80)$.

\section{Resultados}

La Tabla 2 recoge las medias, desviaciones típicas, significación y tamaño del efecto de las comparaciones entre el pretest y el post-test en ambos grupos.

En la fase pretest, los resultados muestran que no existieron diferencias estadísticamente significativas en las puntuaciones en las diferentes variables entre el grupo experimental y el grupo control. La única excepción fue la variable Fobia Social, para la cual el grupo control mostró mayores puntuaciones que el grupo experimental, con un tamaño del efecto entre pequeño y mediano.

Al comparar los resultados entre pretest-postest en el grupo control, se observa un aumento en las puntuaciones de las variables de Ansiedad Total (tamaño del efecto pequeño), y un descenso en las dimensiones Autoconciencia y Empatía de la Inteligencia Emocional (tamaños del efecto grande y mediano, respectivamente).

Respecto a la comparación pretest-postest en el grupo experimental, hubo una disminución en las puntuaciones de las variables de Ansiedad (sólo estadísticamente significativas en Ansiedad de Separación, Ansiedad Generalizada y Total) y Depresión, y un incremento en las puntuaciones de las escalas de Inteligencia Emocional. De igual modo se observan mayores puntuaciones en los índices de elecciones y menores en los índices de rechazo en el postest respecto al pretest. En todos los casos los tamaños del efecto fueron pequeños.

Respecto a las diferencias entre el grupo control y el grupo de tratamiento en el postest, cabe mencionar que el grupo experimental mostró puntuaciones menores en el grupo de variables clínicas (Ansiedad y Depresión) y en los índices de rechazo y mayores en las dimensiones de Inteligencia Emocional y los índices de elecciones. No obstante, sólo algunas comparaciones resultaron significativas (véase tabla 2), mostrando en todos los casos tamaños del efecto entre medianos y grandes.

\section{Discusión}

El objetivo de este trabajo fue evaluar la eficacia de un programa de prevención del rechazo social y mejora de las relaciones basado en el desarrollo de competencias sociales e Inteligencia Emocional, con el fin de mejorar las relaciones entre iguales en alumnos de segundo y tercer ciclo de Educación Primaria, y servir como método de prevención del rechazo social y las psicopatologías asociadas.

Con respecto a la primera hipótesis, los resultados obtenidos confirman que el grupo experimental y el grupo control fueron equivalentes en el pretest, presentando puntuaciones equivalentes en las escalas de Ansiedad, Depresión, Inteligencia Emocional e Índices de rechazo y elecciones. Estos resultados son similares a los hallados en el estudio de Llanos (2006), en el cual los niños y niñas del grupo control y el grupo experimental presentaban el mismo nivel de socialización, con puntuaciones equivalentes en popularidad, sensibilidad social, ansiedad y apatía.

En relación a la segunda hipótesis, en general los resultados fueron los esperados, al no encontrar cambios significativos entre pretest y postest en el grupo sin tratamiento. Sin embargo dicha hipótesis sólo se confirmó parcialmente, ya que hubo un aumento significativo en la sintomatología ansiosa general y una disminución en la autoconciencia y la empatía (dimensiones de la Inteligencia Emocional) entre pretest y postest en el grupo control. Estas diferencias pueden deberse a las condiciones de evaluación y a la tendencia de los sujetos de contestar los cuestionarios hacia uno de los polos de respuesta. Por su parte, Morgenson, Reidor y Campen (2005) aseguran que la falta de motivación y reforzamiento 
Tabla 2. Medias, desviaciones típicas, significación y tamaño del efecto de las comparaciones entre el pretest y el post-test en ambos grupos.

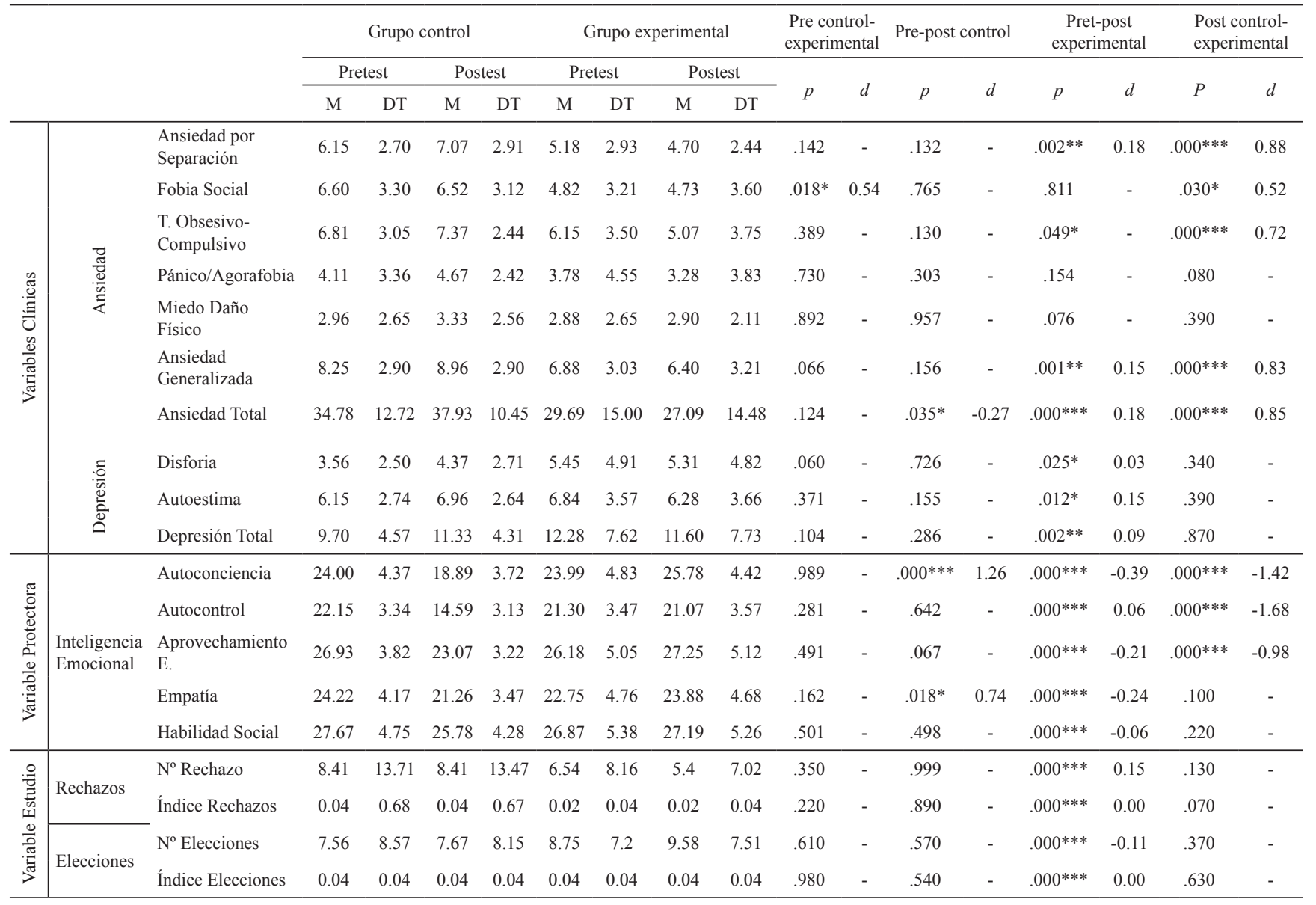

Nota: $* \mathrm{p}<.05 ; * * \mathrm{p}<.005 ; * * * \mathrm{p}<.001$.

en el uso de las habilidades sociales poco a poco lleva a los individuos a la pérdida de conductas habilidosas y una disminución en la socialización.

La tercera hipótesis también se confirma en el sentido de que hay una mejoría entre pretest y postest en el grupo intervenido. Estos cambios se manifiestan en un descenso significativo de las variables de Ansiedad y Depresión, como sucede en el estudio de Llanos (2006), donde el grupo experimental vio reducido su comportamiento en aspectos negativos de la socialización, como apatía, agresividad, ansiedad y timidez. Por otro lado se observan avances significativos entre los beneficiarios del programa en las áreas de la competencia social (Fernández-Cabezas et al., 2011), con un incremento significativo en las escalas de Inteligencia Emocional como autoconciencia, aprovechamiento emocional (automotivación), empatía y habilidad social (Livacic-Rojas, Espinoza y Ugalde, 2004; Pérez et al., 2005), manteniéndose estables los índices de rechazos y elecciones. Resultados similares aparecen en el estudio de Monjas y González (2000) donde los niños del grupo intervenido mostraban más habilidades relacionadas con emociones y sentimientos, y habilidades de solución de problemas interpersonales.

Por último la cuarta hipótesis también se confirma al haber diferencias entre grupo de tratamiento y grupo control en el postest, las puntuaciones en escalas de Ansiedad en el grupo experimental disminuyen, y aumentan las escalas de Inteligencia emocional, esto quiere decir que los niños que participaron en el programa, después de la aplicación presentan menos sintomatología ansiosa, mejores habilidades sociales y mayor empatía, autoconciencia, autocontrol y automotivación. Nuestros resultados coinciden con los hallazgos de Pichardo, García, Justicia y Llanos (2008), puesto que tras la realización del programa de intervención, los niños del grupo experimental en comparación con los del grupo control tenían una mayor sensibilidad social, 
mejor competencia y habilidad social. También Llanos (2006) informó que los niños del grupo experimental tras el tratamiento tenían una mayor capacidad de llevarse bien con sus iguales, habían mejorado sus relaciones sociales, eran más populares y tenían una mayor sensibilidad social que los niños del grupo control a los que no se les aplicó el programa.

Los resultados obtenidos permiten mantener una actitud positiva respecto a las posibilidades que ofrece el programa de desarrollo de competencias socio-emocionales con niños, dado que el grupo experimental y el grupo control fueron equivalentes en el pretest, se hallaron cambios entre las medidas de ciertas variables en el pretest y el postest el grupo control, se produjo una mejoría en las variables consideradas entre pretest y postest en el grupo de intervención, y hubo diferencias entre grupo de tratamiento y grupo control en el postest.

Si bien los resultados obtenidos en esta investigación son satisfactorios, debemos hacer ciertas puntualizaciones al respecto. En primer lugar, este programa fue aplicado a niños entre 8 y 12 años; por lo que no se recomienda extrapolar las mejoras observadas a niños de otras edades. La aplicación del programa a otras edades implicaría una adaptación tanto de las técnicas utilizadas como de las situaciones problema que se plantean, lo que daría paso a otra investigación y a la obtención de nuevos resultados. Segundo, el Test de Inteligencia Emocional presenta alguna dificultad menor en la comprensión del vocabulario por parte de los alumnos, por lo que se recomienda realizar una validación y adaptación del cuestionario para su uso en población española. Tercero, el programa de intervención aplicado a niños se realizó en grupos reducidos en el aula, por lo que sería necesario realizar otras investigaciones que permitiesen comprobar si estos resultados son equiparables a los obtenidos en las intervenciones realizadas de forma individual, atendiendo a las particularidades de cada niño (Cohen, 1995). Cuarto, la muestra de este estudio ha sido reducida, por lo que se recomienda replicar este estudio piloto ampliando el número de participantes en ambos grupos y para que los resultados puedan ser generalizables al resto de la población. Finalmente, es necesario realizar estudios de seguimiento a medio y largo plazo que permitan valorar si las mejoras se mantienen a lo largo del tiempo y se generalizan a diversos contextos, siendo interesante obtener información de los sujetos proporcionada por los padres o profesores mediante la administración de cuestionarios.

En resumen, resulta evidente la necesidad de emplear programas destinados al desarrollo y mejora de las habilidades sociales en niños, la necesidad de desarrollar programas transversales dentro de los contenidos del currículum escolar, además de fomentar y trabajar estrategias adecuadas para el comportamiento social de los alumnos en sus respectivas aulas (Díaz-Barriga y Hernández, 2001), para prevenir la aparición del rechazo social en edades más tempranas y patologías asociadas a este.

\section{Referencias}

Asher, S., y Paquette, J. A. (2003). Loneliness and peer relations in childhood. Current Directions in Psychological Science, 12, 75-78.

Bierman, K. L. (2004). Peer rejection. Developmental, processes and intervention strategies. Nueva York, EE.UU: The Guilford Press.

Bukowski, W. M., Newcomb, A. F., y Hartup, W. W. (1996). The company they keep: Friendship in childhood and adolescence. Nueva York, EE.UU: Cambridge University Press.

Campbell, W. K, Krusemark, E. A., Dyckman, K. A., Brunell, A. B., McDowell, J. E., Twenge J. M., y Clementz, B. A. (2006). A magnetoencephalography investigation of neural correlates for social exclusion and self-control. Social Neuroscience, 1, 124-134.

Chiriboga, R. D., y Franco, J. E. (2001). Validación de un test de Inteligencia Emocional en niños de diez años de edad. Revista Médico de Familia, 9, 24-38.

Cohen, D. J. (1995) Psychosocial therapies for children and adolescents: Overview and future directions. Journal of Abnormal Child Psychology, 23, 141-157.

Cohen, J. (1988). Statistical power analysis for the behavioral science (2nd ed.). Hillsdale, NJ, USA: Lawrence Erlbaum Associates.

Del Barrio, V., Moreno-Rosset, C., y López-Martínez, R. (1999). The Children's Depression Inventory, (CDI; Kovacs, 1992). Su aplicación en población española. Revista Clínica y Salud, 10, 393-416.

Díaz-Aguado, M. (1995). Niños con dificultades socioemocionales. Instrumentos de evaluación. Madrid: Ministerio de Asuntos Sociales.

Díaz-Aguado, M. (2002). Prevenir la violencia contra las mujeres: construyendo la igualdad. Programa para educación secundaria. Madrid, España: Instituto de la Mujer.

Díaz-Barriga, F., y Hernández, G. (2001). Estrategias docentes para un aprendizaje significativo. México DF: McGraw-Hill.

Eshel, Y., Sharabany, R, y Barsade, E. (2003) Reciprocated and unreciprocated dyadic peer preferences and academic achievement of Israeli and immigrant students: a longitudinal study. Journal of Social Psychology, $143,746-762$.

Fernández-Cabezas, M., Benítez, J. L., Fernández, E., Justicia, F., y Justicia-Arráez, A. (2011). Desarrollo de la competencia social y prevención del comportamiento antisocial en niños de 3 años. Infancia y Aprendizaje, 34, 337-347.

Figueras, A., Amador-Campos, J. A., Gómez-Benito, J., y del Barrio, V. (2010) Propiedades psicométricas del Inventario de Depresión Infantil en una muestra comunitaria y clínica. The Spanish Journal of Psycho$\log y, 12,990-999$.

García-Bacete, F. J., Monjas, I., y Sureda, M. I. (2008). Distribución sociométrica en las aulas de chicos y chicas a lo largo de la escolaridad. Revista de Psicología Social, 23, 63-74.

García-Bacete, F. J., Monjas, I., y Sureda, M. I. (2010). El rechazo entre iguales en la educación primaria: una panorámica general. Anales de Psicología, 26, 123-136.

Gifford-Smith, M. E., y Brownell, C. A. (2003). Childhood peer relationships: Social acceptance, friendships, and peer networks. Journal of School Psychology, 41, 235-284.

Guerra, C., Campaña, M.A., Fredes, V., Gutiérrez, L., y Plaza, H. (2011). Regulación de la Agresividad Entre Preescolares Mediante el Entrenamiento a Madres y Profesoras. Terapia Psicológica, 29, 197-211. 
Herrera, E., Núñez, A.C, Tobón, S., y Arias, D. (2009). Análisis bibliométrico de la depresión infantil. Pensamiento Psicológico, 5, 59-70.

Hussong, A. M. (2000). Perceived peer context and adolescent adjustment. Journal of Research on Adolescence, 10, 391-415.

Inglés, C.J., Benavides, G., Redondo, J., García-Fernández, J.M., RuizEsteban, C., Estévez, C., y Huescar, E. (2009). Conducta prosocial y rendimiento académico en estudiantes españoles de Educación Secundaria Obligatoria. Anales de Psicología, 25, 93-101.

Inglés, C.J., Delgado, B., García-Fernández, J.M., Ruíz-Esteban, C., y Díaz-Herrero, A. (2010). Sociometric types and social interaction styles in a sample of Spanish Adolescents. The Spanish Journal of Psychology, 13, 730-740.

Knut, G., y Frode, S. (2005). Evaluation of a Norwegian postgraduate training programme for the implementation of Aggression Replacement Training. Psychology, Crime \& Law, 11, 435- 444.

Livacic-Rojas, P., Espinoza, M., y Ugalde, F. (2004). Intervenciones psicológicas basadas en la evidencia para la prevención de la delincuencia juvenil. Un programa de habilidades sociales en ambientes educativos. Terapia Psicológica, 1, 83-91.

Llanos, C. (2006). Efectos de un programa de entrenamiento en habilidades sociales. (Tesis Doctoral). Departamento de Psicología Evolutiva y de la Educación. Facultad de Ciencias de la Educación. Granada.

Magallares, A. (2011). Exclusión social, rechazo y obstracismo: principales efectos. Psicología.com, 15. Recuperado desde http://hdl.handle. net/10401/4321

Mckown, C. (2010). Tres motivos para el rechazo social. Kindsein.com. Recuperado desde http://kindsein.com/es/33/2/775/

Mestre, V., Samper, P., y Frías, M. D. (2004). Personalidad y contexto familiar como factores predictores de la disposición prosocial y antisocial de los adolescentes. Revista Latinoamericana de Psicología, 36, 445-457.

Monjas, M. I., y González, B. (Dirs.) (2000). Las habilidades sociales en el currículo. Madrid: Ministerio de Educación y Cultura. Centro de Investigación y Documentación Educativa (CIDE).

Morgerson, M., Reider, T., y Campen, C. (2005). Selecting individuals in team settings: the importance of social skills, personality characteristics, and teamwork knowledge. Personnel Psychology, 58, 583-611.

Muñoz, V., (2007). El rechazo entre iguales en el ámbito educativo. Infocop online. Recuperado desde http://www.infocop.es/view_article. asp?id=1599
Orgilés, M. (2009). Versión española de la Escala de Ansiedad Infantil de Spence (Spence Children's Anxiety Scale; SCAS). Recuperado desde http://www.scaswebsite.com/docs/spanish-euro-scas.pdf

Orgilés, M., Méndez, X., Spence, S. H., Huedo-Medina, T. B., y Espada, J. P. (2012). Spanish validation of the Spence Children's Anxiety Scale. Child Psychiatry and Human Development, 43, 271-281.

Pérez, V., Fernández, A. M., Rodríguez, J., y de la Barra, F. (2005). Evaluación del Efecto de una Intervención Conductual en la Agresividad Escolar. Terapia Psicológica, 1, 91-98.

Pichardo, M. C., García, T., Justicia, F., y Llanos, C. (2008). Efectos de un programa de intervención para la mejora de la competencia social en niños de educación primaria en Bolivia. International Journal of Psychology and Psychological Therapy, 8, 441-452.

Plazas, E. A., Morón, M., Sarmiento, H., Ariza, S., Santiago, A., y Darío, C. (2010). Relaciones entre iguales, conducta prosocial y género desde la educación primaria hasta la universitaria en Colombia. Universitas Psychologica, 9, 357-369.

Povedano, A., Estévez, E., Martínez, B., y Monreal, M.C. (2012). Un perfil psicosocial de adolescentes agresores y víctimas en la escuela: análisis de las diferencias de género. Revista de Psicología Social, 27, 169-182.

Rajmil, L, Alonso, J., Analitis, F., Detmar, S., Erhart, M., Klein, M., Ravens-Sieberer, U., Herdman, M., Berra, S., y European Kidscreen Group (2009). Víctimas de acoso: factores asociados en niños y adolescentes de 8-18 años de edad en 11 países europeos. Pediatrics (Ed esp), 67, 111-118.

Ramos, J. L. (2011). Investigación cuasiexperimental. En S. Cubo, B. Martín y J. L. Ramos (Coords.), Métodos de investigación y análisis de datos en ciencias sociales y de la salud (pp. 329-371). Madrid: Pirámide.

SPSS, 11.0 (2001). Statistical Package for the Social Sciences. SPSS Inc. Wentzel, K. R. (2003). Sociometric status and adjustment in middle school: A longitudinal study. Journal of Early Adolescence, 23, 5-28.

Yugar, J. M., y Shapiro, E. S. (2001). Elementary children's school friendship: A comparison of peer assessment methodologies. School Psychology Review, 30, 568-585.

Zhu, L., Xu, Zh., y Kong, R. (2000) A study of attention, behaviour characteristics, and peer relations in children with learning disabilities. Psichological Science (China), 23, 556-559. 
